1

\title{
Drivers anticipate lead-vehicle conflicts during automated longitudinal control: sensory cues capture driver attention and promote appropriate and timely responses
}

\author{
Alberto Morando ${ }^{\mathrm{a}, *}$, Trent Victor ${ }^{\mathrm{a}, \mathrm{b}}$, and Marco Dozza ${ }^{\mathrm{a}}$ \\ ${ }^{a}$ CHALMERS - University of Technology, Department of Applied Mechanics, Division of Vehicle Safety, Sweden \\ ${ }^{b}$ Volvo Car Safety Centre - Volvo Car Corporation, Sweden \\ * Correspondence address: SAFER, Box 8077, 40278 Göteborg, Sweden \\ Tel.: +4670 3733694 \\ E-mail address: alberto.morando@chalmers.se
}

Abstract Adaptive Cruise Control (ACC) has been shown to reduce the exposure to critical situations by maintaining a safe speed and headway. It has also been shown that drivers adapt their visual behavior in response to the driving task demand with ACC, anticipating an impending lead vehicle conflict by directing their eyes to the forward path before a situation becomes critical. The purpose of this paper is to identify the causes related to this anticipatory mechanism, by investigating drivers' visual behavior while driving with ACC when a potential critical situation is encountered, identified as a forward collision warning (FCW) onset (including false positive warnings). This paper discusses how sensory cues capture attention to the forward path in anticipation of the FCW onset. The analysis used the naturalistic database EuroFOT to examine visual behavior with respect to two manually-coded metrics, glance location and glance eccentricity, and then related the findings to vehicle data (such as speed, acceleration, and radar information). Three sensory cues (longitudinal deceleration, looming, and brake lights) were found to be relevant for capturing driver attention and increase glances to the forward path in anticipation of the threat; the deceleration cue seems to be dominant. The results also show that the FCW acts as an effective attention-orienting mechanism when no threat anticipation is present. These findings, relevant to the study of automation, provide additional information about drivers' response to potential lead-vehicle conflicts when longitudinal control is automated. Moreover, these results suggest that sensory cues are important for alerting drivers to an impending critical situation, allowing for a prompt reaction.

Keywords: naturalistic driving, glance analysis, automation, driver behavior, forward collision warning, adaptive cruise control. 


\section{INTRODUCTION}

Adaptive cruise control (ACC) is an advanced driver assistance system (ADAS) that automates the longitudinal control of the vehicle. This system, classified as level 1 automation (NHTSA, 2015; SAE, 2014), maintains speed and time headway according to chosen settings. The driver activates and sets the ACC system by pressing buttons on the steering wheel. When a lead vehicle is detected, the speed is automatically controlled to keep the selected headway. However, ACC's braking capacity is limited to a level sufficient for normal headway maintenance situations, not extreme braking situations. The allowed deceleration varies among implementations, but the ACC maximum braking authority is usually about 0.3g, as suggested in the standards ISO 15622:2010 and ISO 22179:2009. When the driving situation exceeds the braking capacity of the ACC, because of a highly decelerating lead vehicle, for example, a frontal collision warning ( $\mathrm{FCW}$ ) is issued. The FCW's role is to redirect the driver's attention to the forward road and elicit a driver braking response in critical situations, by means of visual and auditory signals. ACC has primarily been seen as a system supporting normal driving situations, for comfort. However, by maintaining a safe speed and headway, ACC and FCW have been shown to improve safety-related measures, reducing the exposure to critical situations (Malta et al., 2011; NHTSA, 2005).

Based on the hierarchical structure proposed by Michon (1985), ACC primarily supports the driver at the control level (i.e. accelerating and braking) and the maneuvering level (i.e. speed selection, gap acceptance and obstacle avoidance); it does not perform the entire dynamic driving task. The driver must monitor the system and take over when required, either by the system itself (e.g., when a FCW is issued) or when ACC does not react to a lead vehicle due to system limitations, such as the radar's field-ofview. Several studies questioned the ability of a driver to reclaim control in an effective and safe manner after a system failure. They raised concerns about the harmful effect of ACC (and, by extension, of higher levels of automation) due to the degradation of situation awareness and a slower response to critical events (for example); for a review see de Winter et al. (2014). Situation awareness is defined as "the perception of the elements in the environment within a volume of time and space, the comprehension of their meaning, and the projection of their status in the near future" (Endsley, 1988, p. 792). The review by de Winter et al. (2014) shows that results for situation awareness vary between studies. ACC use can result in deteriorated situation awareness when drivers engage in secondary tasks, but improves situation awareness if they are attending to the driving task. Similarly, a number of experiments have found that ACC drivers can be slower to respond to critical events compared to manual drivers, while many studies have shown faster reactions to artificial visual stimuli (de Winter et al., 2014). A more nuanced examination of the response processes in critical events when using ACC is clearly needed.

A possible explanation for degraded detection of and response to critical driving situations can be regarded as an unintended effect, also known as behavioral adaptation (OECD, 1990). For example, 
ACC decreases the visual demand of driving; as a consequence drivers use freed resources to engage in non-driving activities, which may reduce the attention allocated for monitoring the road ahead (RudinBrown \& Parker, 2004). The widespread availability of in-vehicle infotainment systems and nomadic devices may further aggravate this effect (Lee et al., 2006). In their naturalistic study, Malta et al. (2011) found a general increase in secondary-task engagement while driving with ACC. A follow-up study by Tivesten et al. (2015) examined the drivers' visual attention in motorway car-following scenarios. In steady state driving, the analysis confirmed a lower attention level to the forward path with ACC than without ( $77 \%$ mean eyes on path with ACC, compared to $\sim 85 \%$ for manual driving without ACC). Tivesten et al (2015) also clarified that most of the glances away from the forward path were drivingrelated. Because driving relies heavily on vision (Shinar, 2007), diversion of visual attention from the forward road could lead to a collision there. However, Malta et al. (2011) pointed out that drivers kept their attention on the primary driving task in critical situations. Furthermore, Tivesten et al. (2015) showed a threat anticipation response: drivers anticipate the impending criticality by directing their eyes to the forward roadway before a situation becomes critical. This is evidence that allocation of attention away from the road is a function of the current driving situation demand (Ranney, 1994; Summala, 2007).

A simulator study by Lee et al. (2006) evaluated the effectiveness of warning modalities at reengaging drivers when the ACC capabilities are exceeded. Their results showed that if warned that an intervention is needed, drivers could effectively resume control even if distracted. However, other studies showed that drivers responded poorly to unexpected events or failures for which alerts are not provided-for example, sensor failures (Nilsson et al., 2013; Rudin-Brown \& Parker, 2004; Stanton et al., 1997; Strand et al., 2014). Fortunately, in the real world these failures are rare, thanks to technology advances and sensor redundancy; even so, providing feedback on the system status and availability is recommended by the standard ISO 15622:2010. Therefore, the difficulties encountered by drivers may be overrepresented in studies when such feedback is not provided (Lee et al., 2006).

Although the FCW is intended to redirect the gaze of the driver towards the forward path and inform the driver that an avoidance maneuver is needed, the results in (Tivesten et al., 2015) suggested that there may be other cues that elicit a shift of visual attention in anticipation of a critical situation, even before an FCW is issued. However, the cause for this anticipatory mechanism was not clearly identified; hence the need for further investigation. Tivesten et al. (2015) showed that the average percent of eyes on path increased steadily over time, and they suggested that this increase was due to drivers' reactions to external stimuli (e.g., related to the approach toward the lead vehicle).

This study discusses three sensory cues which are considered relevant for prompting the drivers' visual attention towards the forward path in anticipation of a lead vehicle conflict. The first cue is the detection of the longitudinal acceleration of the driver vehicle by the vestibular system. As pointed out in (Lee et al., 2006; 2007), another benefit of the ACC is that the cue associated with the speed modulation 
(deceleration or braking) before the onset of the warning may be particularly effective at alerting drivers and making them resume control when needed. Subjective data from a field operational test of ACC (Fancher et al., 1998) indicated that drivers acknowledged the deceleration cue as beneficial for informing them of an evolving headway conflict. Lee et al. (2006) found the detection threshold to be between $0.15-0.20 \mathrm{~m} / \mathrm{s}^{2}$. However, this deceleration cue effect is often discounted in studies in fixedbase simulators, since they do not provide these deceleration cues. The second cue is visual looming, the optical expansion of the lead vehicle in the eye of the driver. Visual cues have been shown to be particularly relevant in car-following scenarios. Previous studies have argued that the driver could detect changes in relative velocity and control the evasive maneuvers (e.g., braking) based solely on information like the visual angle subtended by the lead vehicle $(\theta)$, the rate of change $(\dot{\theta})$, or the combination thereof ( $\tau$ ) (See, for example, Hoffmann, 1968; Hoffmann \& Mortimer, 1994a; Lee, 1976; Mortimer, 1990). More details on these measures are given in section 2.5.

116 Visual detection performance generally deteriorates towards the retinal periphery, therefore the further 117 the driver diverts the eyes away from the forward path the worse the ability to detect threats and objects 118 on the road (Victor et al., 2008). However, results from laboratory experiments show that certain salient stimuli (e.g., moving and looming targets) induce automatic and reflexive reactions. When one of these stimuli occurs, the attention is shifted to the stimulus, especially when it is not expected (Jonides, 1981; Klein et al., 1992; Regan \& Vincent, 1995). The salient stimuli expected to elicit an attention shift are associated with behavioral urgency. For example, given stimuli of the same magnitude, looming objects indicate an impending collision and would trigger a reflexive response, whereas receding objects should not elicit the same response, being neither potentially urgent nor threatening (behavioral urgency hypothesis in Franconeri \& Simons, 2003; Lin et al., 2008). In on-road studies, drivers could detect a closing car even when visual attention was diverted away from the road, but with increasing eccentricity the threshold for detection increased (Lamble et al., 1999; Summala et al., 1998). (The table in Appendix B provides a compilation of the results from these two studies.) When looking along the line of motion, the perceptual threshold of $\dot{\theta}$ for discriminating the closure of the lead vehicle was around $0.0036 \mathrm{rad} / \mathrm{s}$

130 (with a minimum value of $0.0022 \mathrm{rad} / \mathrm{s}$ ), regardless of the test conditions (initial headway, speed, and deceleration). This threshold is higher than, yet comparable to, the value of about $0.0030 \mathrm{rad} / \mathrm{s}$, which was proposed by studies from experiments in which the participants were required to watch film clips, and from reviews of previous findings (Hoffmann \& Mortimer, 1994a; Hoffmann \& Mortimer, 1994b;

134 Mortimer, 1990). With increasing eccentricity, the detection threshold for $\dot{\theta}$ increases linearly.

135 However, there is little agreement on the results for $\tau^{-1}$ since, unlike $\dot{\theta}$, this variable may be quite sensitive to the different experimental conditions.

137 The third cue is the brake light onset. The brake light onset signals that the lead vehicle started braking, but its predictive value is limited and it does not give information about the criticality of the situation, e.g., whether/how hard one must brake (Lee, 1976). In their study of naturalistic crashes and near- 
crashes, Victor et al. (2015) concluded that brake lights had a limited impact on driver behavior in rearend situations. In fact, the brake light onsets which occurred while the driver was looking forward were generally ignored (i.e. the drivers were willing to take their eyes off path while the brake lights were still illuminated) and do not seem to have notably influenced the driver reaction. One explanation is that, in real world driving, drivers may be exposed to brake light onsets which are not associated to any threat, leading to a cry-wolf effect (Victor et al., 2015). Furthermore, Markkula et al. (2016) and Victor et al. (2015) showed that reaction times in real crashes and near-crashes are influenced by lead-vehicle looming, and not by brake light onsets as reported, for example, by Young and Stanton (2007). Assuming that the brake lights are salient enough to be detected while looking ahead (consider, for example, the difficulty encountered in strong sunlight), Summala et al. (1998) found that detection was significantly impaired in the periphery, even at a low level of eccentricity ${ }^{1}$. However, for night driving, the stimulus would be more salient and might be more easily detected, making the change in angular separation of the car's brake lights the prominent cue in the detection of relative speed (Janssen (1974).

There are a multitude of other attentional capture cues that were not taken into account in this study. Such cues might be related to the road infrastructure (e.g., road signs), to the surrounding traffic (e.g., behavior of other vehicles), to other visual properties (e.g., color, luminance, and contrast), and to cognitive and motivational factors (e.g., experience).

In summary, the present study investigates visual behavior when potentially critical situations (identified as FCW onsets) are encountered while driving with ACC and identifies possible reasons for the anticipatory response. The main hypothesis was that visual and vestibular/somatosensory cues were responsible for orienting the drivers' visual attention towards the forward path in anticipation of the FCW onset.

\section{METHODS}

\subsection{Data source}

The data used in this study are from the Swedish subset of the EuroFOT database, collected from 100 Volvo cars (2009-2010 V70 and XC70 models) in the region of Västra Götaland over the period 2010-2011. EuroFOT (Kessler et al., 2012) was a Field Operational Test sponsored by the European Community to evaluate the impact of ADAS on routine driving in real traffic. Among the ADAS tested, the ones of particular interest for this study were the ACC and the FCW. All 263 drivers who participated in the project were Volvo employees who volunteered to participate in the study and drove their own cars.

\footnotetext{
${ }^{1}$ In (Summala et al., 1998) an old compact car was used (1988 Lada Samara), and it is not clear if it was equipped with a center high-mount stop lamp (CHMSL) which could have improved the detection of the brake light onset.
} 
171 Data were continuously collected from the controller area network (CAN) bus, from extra sensors (e.g.,

172 accelerometer, GPS), and from cameras mounted in the cars, sampled at $10 \mathrm{~Hz}$. The collection began

173 when the engine was started, and it was interrupted when the ignition was turned off. The driver inputs

174 were gathered both from the CAN bus (i.e. pedals and steering wheel activity) and a camera recording

175 foot movement. Other cameras were used to record the forward and backward view of the vehicle and

176 the face of the driver. All CAN signals were pre-processed (e.g., decoded, synchronized, filtered) and

177 stored in a relational database.

\section{$178 \quad 2.2$ Event dataset}

179 Initially, 280 critical events were extracted from the database. A critical event occurred any time a FCW 180 was issued while driving with ACC. A critical event consisted of $20 \mathrm{~s}$ of driving, centered at the FCW 181 onset (i.e. $10 \mathrm{~s}$ before and after the warning). General inclusion criteria were that before and at the onset of FCW, the ACC and FCW were active (the ACC was active at speeds above $30 \mathrm{~km} / \mathrm{h}$ and disengaged

183 when the driver pressed the brake pedal) and the alert modality of the FCW was visual and audio 184 (according to the specifications described in Coelingh et al., 2007). The events were reviewed, and 185 discarded if the video sources were not available or the driver's eyes were not clearly visible. Events 186 were also rejected if, in the $5 \mathrm{~s}$ interval before the FCW, the driver changed the ACC or the FCW settings 187 (e.g. ACC set speed, FCW sensitivity, etc.), changed lanes or overtook another vehicle, or overrode the 188 ACC by accelerating (i.e. the driver intentionally pursued a small forward headway to the lead vehicle, 189 making the warning predictable). Finally, events were also discarded if a lane departure warning (LDW) 190 was triggered prior to the onset of FCW; otherwise the LDW may have confounded the effect of the 191 FCW.

192 In the end, 125 events fulfilled the inclusion criteria and were included in the analysis. The dataset 193 included 43 unique drivers (36 males and 7 females) between the ages of 18 and 61 (Mean $=47.76$ 194 years, standard deviation $=9.13)$. 


\subsection{Annotated variables}

196

197

198

199

200

201

202

203

204

205

206

207

208

209

210

211

212

213

214

215

216

217

218

219

\subsubsection{Event classification}

Each event was categorized as either rear-end FCW or random FCW. An event was classified as rearend FCW if the FCW was due to an imminent collision with a lead vehicle (from now on referred as principal other vehicle, POV). An event was classified as random FCW if a FCW was issued but no POV (or other threat) was present. A random FCW was probably the consequence of limitations in processing the radar information or in detection. A total of 87 rear-end FCW and 38 random FCW were analyzed.

\subsubsection{Glance coding}

In order to study the drivers' visual behavior two metrics were used: glance location and glance eccentricity. Glance location is the area of interest (AOI) the eyes are directed to. This metric identifies where the drivers were looking, in order to quantify how they allocated their visual attention; looking towards the road center is primary to safe maneuvering of the vehicle, although they also performed secondary visual tasks. Secondary tasks can be either driving-related (e.g. reading road signs, checking speedometer) or not (e.g. phone-related, reaching for objects). The glance location was manually coded frame by frame, based on the forward and driver videos (recorded at 10 frames per second), with the support of the MATLAB-based program FOTware (Dozza et al., 2010). The coding was in line with the work performed in (Tivesten et al., 2015; Victor et al., 2015). The main AOIs are illustrated in Figure 1 and a more complete description is given in Table A.1. Glance eccentricity is defined as the radial angle between the forward path and the glance location. Inspired by the work done in (Klauer et al., 2006, p. 107), the annotated AOIs were grouped into four levels based on the average angle away from the forward path (see Table A.2 for further details).

\subsubsection{Other annotations}

Relevant timestamps were annotated (i.e. the time of FCW onset and the time when the POV's brake lights turned on) as well as information about lighting, weather conditions, and road geometry (see Table

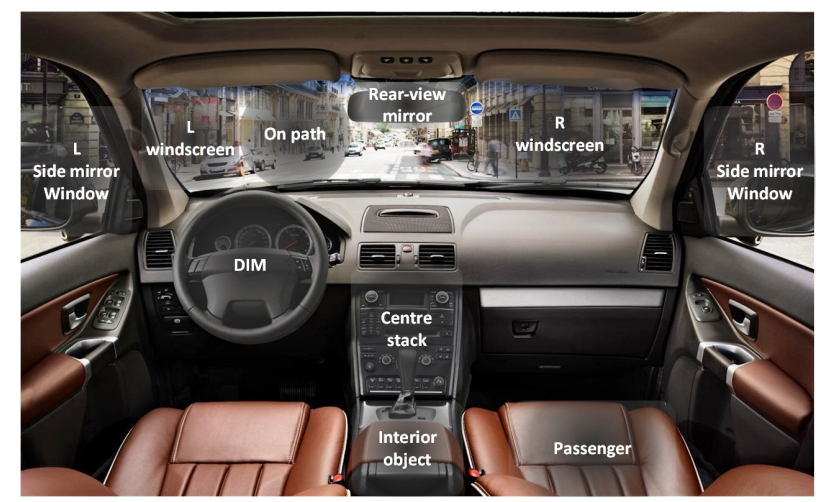

Figure 1. Location of the main AOls. Background photo: Volvo car. 
A.3 for a summary of the annotated environmental variables). Moreover, a narrative description of each event was written.

\subsection{Vehicle measures}

Among the signals collected from the CAN bus, the ones of particular interest for describing the vehicle dynamics and the driving situation (specifically the interaction with the POV) were the speed and the longitudinal acceleration of the driver vehicle, the distance (Range) and the relative speed (Range rate) to the POV, the time to collision (TTC), and the time headway (THW). The TTC and THW are two commonly used safety indicators. TTC is computed as the ratio between the distance and the relative speed to the POV; it expresses the severity of the impending collision. In this study the inverse of the TTC $\left(\mathrm{TTC}^{-1}\right)$ was used in order to have a measure that increases as the risk of colliding with the POV increases (Summala, 2000, defined it as urgency). THW is computed as the ratio between the distance to the POV and the speed of the driver vehicle. The THW, in steady-state car following, is a measure of the exposure to a potential threat. For example, if the leading vehicle brakes, a short THW would require a faster response than a longer THW in order to avoid the collision. It is important to remember that in the events under analysis, the drivers selected the desired THW to maintain in the ACC settings.

\subsection{Optical variables}

Three optically-defined variables were used: theta $(\theta)$, its time derivative theta $\operatorname{dot}(\dot{\theta})$, and their ratio $\tan (\tau)$. The angle $\theta$ is the optical angle (in rad) of the POV at the eyes of the driver ${ }^{2}$ and it is calculated by

$$
\theta=2 \cdot \operatorname{atan} \frac{W}{2 R}
$$

where $W$ is an estimation of the POV width (a standard value of $1.8 \mathrm{~m}$ was used, see also Victor et al., 2015, p. 28), and $R$ is the distance to the POV (in $\mathrm{m}$ ). The rate of change of the optical angle, $\dot{\theta}$ (in $\mathrm{rad} / \mathrm{s})$, is calculated by

$$
\dot{\theta}=-\frac{4 W R}{R^{2}+4 W^{2}}
$$

where $\dot{R}$ is the relative speed of the driver vehicle (in $\mathrm{m} / \mathrm{s}$ ) and the POV. Finally, $\tau$ is given by the ratio of $\theta$ and $\dot{\theta}$, that is the rate of dilation of the retina image of the POV (in s) as proposed in (Lee, 1976). As in the case of TTC, in the analysis the inverse of $\tau\left(\tau^{-1}\right)$ was used instead. It also turns out that $\tau$ is the optical approximation of the physical quantity TTC (Lee, 1976).

\footnotetext{
${ }^{2}$ Properly speaking, the angle $\theta$ is estimated based on information from the front radar, therefore it is the optical angle at the bumper of the driver vehicle.
} 


\subsection{Data analysis framework}

\subsubsection{Response and explanatory variables}

In the analysis framework of this study, the response variable is the time-course of the driver's visual behavior. In order to visualize it, a stacked histogram was used (see an example in Figure 2, and refer to Appendix A for the glance's color code). This graph is inspired by previous studies (Tivesten et al., 2015; Victor et al., 2015) and shows the proportion of various glances over time in the events under analysis. In addition, the curve for the mean percentage of eyes on path (\%EOPmean) was used to better visualize the trend of the glances on path across the events (black curve in Figure 2). In line with the work done in (Tivesten et al., 2015), the curve was defined by chunking the glance time series of the events in bins of $0.5 \mathrm{~s}$. Each point in the curve is defined by first computing the mean percentage of glances on path in the selected bin for each single event. Then the computed values are averaged across all the events. The errors bars indicate the $95 \%$ confidence interval $(95 \mathrm{CI})$, which is also a measure of the events' inter-variability. The boundaries of the confidence interval are the value of \%EOPmean in the selected bin plus/minus 1.96 times the standard error of the mean.

The exploratory variables are the measures related to the driver vehicle and the POV, as introduced in sections 2.4-2.5. In order to facilitate the comparison to the response variable (i.e. the drivers' visual behavior) the same approach used to define the \%EOPmean was taken.

\subsubsection{Statistics}

The non-parametric Friedman test and the Wilcoxon signed rank post-hoc test were used to compare the observations within the glance time-series at different points in time during the events. The significance level was set at 0.05 (with the Holm-Šidák correction for multiple comparisons).

\section{RESULTS}

\subsection{Glance location}

\subsubsection{Rear-end FCW events}

Figure 3a shows the glance location history for the rear-end FCW events, centered at the FCW onset. In general, the drivers' visual attention in the $20 \mathrm{~s}$ interval was directed towards the forward path (78.4\%). Most of the off path glances were driving-related. The driver information module accounted for $29.3 \%$, followed by $\mathrm{L}+\mathrm{R}$ windscreen (24.8\%), rear view mirror (14.7\%), and $\mathrm{L}+\mathrm{R}$ side mirror/window (11.9\%).

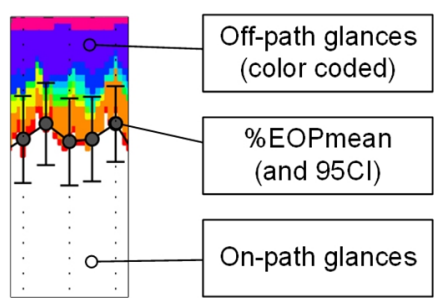

Figure 2. Example of the graph used for visualizing the time-course of the glance location across the events. The graph shows the percentage of glance locations at any given time during the events under analysis. For details on the glances' color code see Appendix A. The black curve indicates the mean percentage of eyes on path (\%EOPmean). The error bars indicate the $95 \%$ confidence interval. 
The cumulative distributions show a noticeable increase in glances towards the rear-view mirror just after the FCW onset (Figure 3b). Moreover, they show that the majority of the glances towards an interior object and the phone were at the beginning and at the end of the events. In contrast, the cumulative distributions of the glances off path towards the other AOIs steadily increased over time.

Based on a graphical analysis of the \%EOPmean trend, five intervals could be identified (Figure 3a):

1. In the $-9.5 \mathrm{~s}--2.5 \mathrm{~s}$ interval the \%EOPmean oscillated around the value $78 \%$, which is in accordance with the baseline used in (Tivesten et al., 2015). Thus, this interval may be defined as the steady state driving interval.

2. In the $-2 \mathrm{~s}-0 \mathrm{~s}$ interval the $\% \mathrm{EOPmean}$ steadily increased from $80.7 \%$ to $95.4 \%$ (significant: Friedman test, $\chi^{2}(2)=44.09, p<.001$; Wilcoxon post-hoc, $z=3.35, p<.01, r=0.25$ ). This interval may be defined as the threat anticipation interval, in conformity with (Tivesten et al., 2015).

3. After the FCW onset, at $0.5 \mathrm{~s}$, the $\% \mathrm{EOPmean}$ further increased to $97.5 \%$. This interval may be defined as the threat interval, as proposed in (Wege et al., 2013).

4. Afterwards, in the $1 \mathrm{~s}-6 \mathrm{~s}$ interval the \%EOPmean decreased to $61.6 \%$, a level that was significantly lower than the beginning of the threat anticipation interval (Friedman test, $\chi^{2}(2)=$ 44.09, $p<.001$; Wilcoxon post-hoc, $z=2.99, p<.01, r=0.23$ ). This interval may be defined as the post-threat interval, as suggested in (Wege et al., 2013).

5. Finally, in the $6.5 \mathrm{~s}-9.5 \mathrm{~s}$ interval the \%EOPmean converged to a value around $70.7 \%$. This interval may be defined as the (back to) steady state driving interval.

\subsubsection{Random FCW events}

Figure 4a shows the glance location history for the random FCW events, centered at the FCW onset. As in the rear-end FCW events, in the $20 \mathrm{~s}$ interval the drivers looked at the forward path most $(78.1 \%)$ of the time. Likewise, most of the glances off path were driving-related. Among them, the driver information module accounted for $34.2 \%$, followed by the rear view mirror $(17.9 \%), \mathrm{L}+\mathrm{R}$ windscreen (15.2\%) and L+R side mirror/window (13.7\%). In general, the cumulative distributions of the off path glances steadily increased over time. In particular, the rapid increase of glances towards the rear-view mirror after the FCW onset, found in the rear-end FCW events, was not present here (Figure 4b).

In Figure 4a, four intervals can be distinguished in the \%EOPmean curve. Note that the threat anticipation interval seen in rear-end events was not present in the random FCW events, but the other four are comparable:

1. In the $-9.5 \mathrm{~s}-0 \mathrm{~s}$ interval the $\% \mathrm{EOPmean}$ oscillated around the value $77.8 \%$. This interval is comparable to the baseline used in (Tivesten et al., 2015) and it may be also defined as the steady state driving interval.

2. After the FCW onset, at $0.5 \mathrm{~s}$, the $\%$ EOPmean suddenly increased from the value of $83.2 \%$ at 


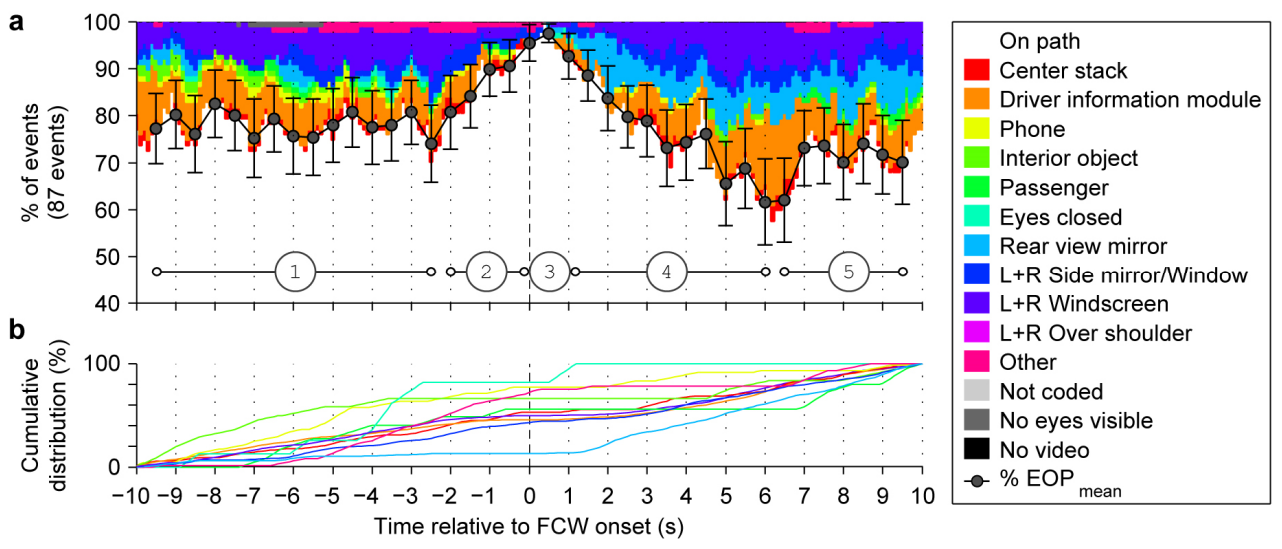

Figure 3. Proportion (a) and cumulative distributions (b) of the glance location over time for the rear-end FCW (frontal collision warning) events. The events are centered at the FCW onset. Numbered circles in (a) indicate the identified intervals described in the text: (1) Steady state driving, (2) Threat anticipation, (3) Threat, (4) Post-threat, (5) (Back to) steady state driving. The curve \%EOPmean shows the mean percentage of eyes on path. The error bars indicate the $95 \%$ confidence interval.

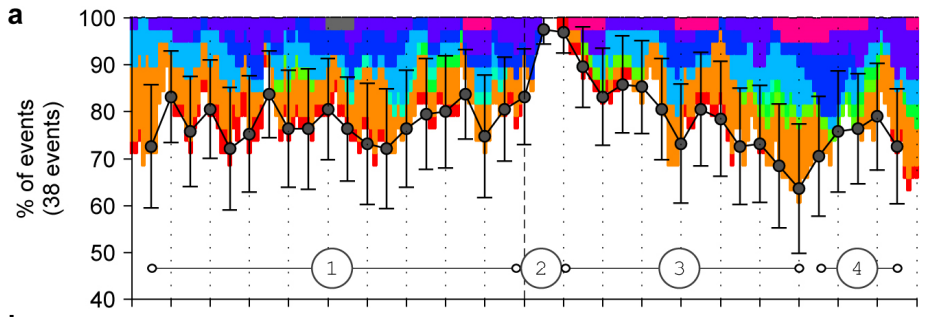

b

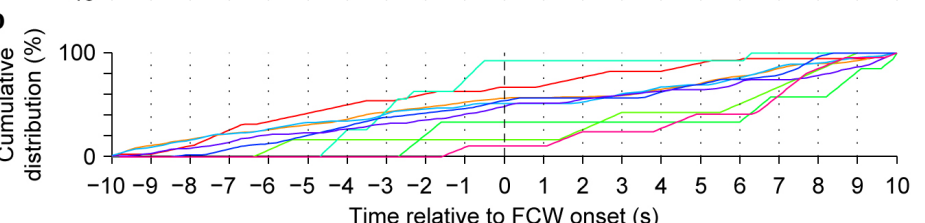

\begin{tabular}{|l|}
\hline On path \\
Center stack \\
Driver information module \\
Phone \\
Interior object \\
Passenger \\
Eyes closed \\
Rear view mirror \\
L+R Side mirror/Window \\
L+R Windscreen \\
L+R Over shoulder \\
Other \\
Not coded \\
No eyes visible \\
No video \\
- \% EOP \\
\hline
\end{tabular}

Figure 4. Proportion (a) and cumulative distributions (b) of the glance location over time for the random FCW (frontal collision warning) events. The events are centered at the FCW onset. Numbered circles in (a) indicate the identified intervals described in the text: (1) Steady state driving, (2) Threat, (3) Postthreat, (4) (Back to) steady state driving. The curve \%EOPmean shows the mean percentage of eyes on path. The error bars indicate the $95 \%$ confidence interval.

the end of the steady state driving interval to $97.4 \%$ (significant: Friedman test, $\chi^{2}(2)=13.23, p$ $<.01$; Wilcoxon post-hoc, $z=2.68, p<.05, r=0.31$ ). This interval may be defined as the threat interval.

3. In the $1 \mathrm{~s}-7 \mathrm{~s}$ interval the $\%$ EOPmean decreased to $63.7 \%$, a level that was significantly lower than the end of the steady state driving interval (Friedman test, $\chi^{2}(2)=13.23, p<.01$; Wilcoxon post-hoc, $z=2.05, p<.05, r=0.24)$. This interval may be defined as the post-threat interval.

4. At the end, in the $7.5 \mathrm{~s}-9.5 \mathrm{~s}$ interval, the $\%$ EOPmean rose to an average value of $74.8 \%$. This interval may be defined as the (back to) steady state driving interval. 


\subsection{Glance eccentricity}

\subsubsection{Rear-end FCW events}

Figure 5a shows the glance history in terms of eccentricity level, time-centered at the FCW onset for the rear-end FCW events. In the $20 \mathrm{~s}$ interval, among the glances away from the forward path, the glances at low eccentricity accounted for $54.1 \%$, whereas the ones at medium and high eccentricity accounted for $27.6 \%$ and $17.8 \%$, respectively. The view of the forward path was thus mostly contained within the near-peripheral visual region (i.e., eccentricity within $30^{\circ}$ ).

In addition to the results described section 3.1.1, it is worth noting that:

- The average percentage of glances at high eccentricity, in the steady state driving and threat anticipation intervals, had a value of $5.1 \%$, which is greater than the average value of $2.7 \%$ in the post-threat and (back to) steady state driving intervals. On the other hand, the opposite held for the glances at medium eccentricity, with an average value of $3.3 \%$ before the threat interval and $9.0 \%$ after. These results are also evident in the cumulative distributions in Figure 5b, which show that the distributions for the glances at high and medium eccentricity were skewed towards the first and second half of the events, respectively.

- The steady increase in \%EOPmean during the threat anticipation interval was primarily a consequence of the steady decrease of glances at low eccentricity, from $8.7 \%$ to $0.2 \%$ (Figure $5 \mathrm{~d})$. Similarly, the steady decrease in \%EOPmean during the post-threat interval was mainly a consequence of the steady increase of glances at low eccentricity, from $3.2 \%$ to $26.2 \%$.

- The median of the distribution of the POV brake onset times across all the events corresponded to the beginning of the threat anticipation interval. The whiskers extended into the $4 \mathrm{~s}$ interval before the FCW onset. Three events were not coded because the POV brake lights did not turn on or were not visible in the video.

\subsubsection{Random FCW events}

Figure 6a shows the glance history in terms of eccentricity level, centered at the FCW onset, for the random FCW events. In the $20 \mathrm{~s}$ interval, among the glances away from the forward path, the glances at low eccentricity accounted for $49.4 \%$, whereas the ones at medium and high eccentricity accounted for $38.6 \%$ and $12 \%$, respectively. As in the rear-end events, the view of the forward path was then mostly contained within the near peripheral region. The cumulative distributions of the glances at low and medium eccentricity steadily increased over time, whereas that of the high-eccentricity glances was slightly skewed towards the end of the events (Figure 6b). 
a

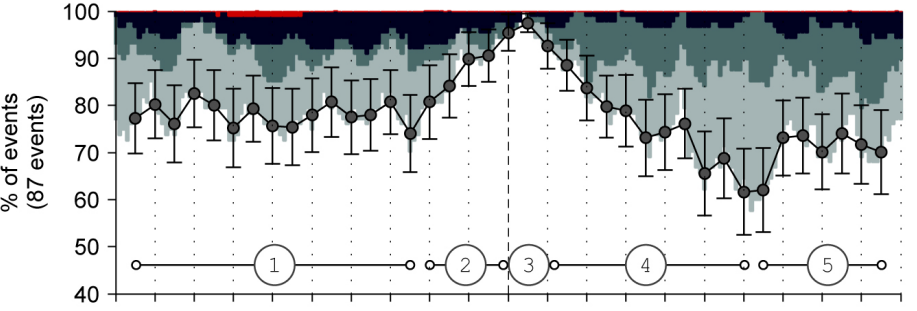

b
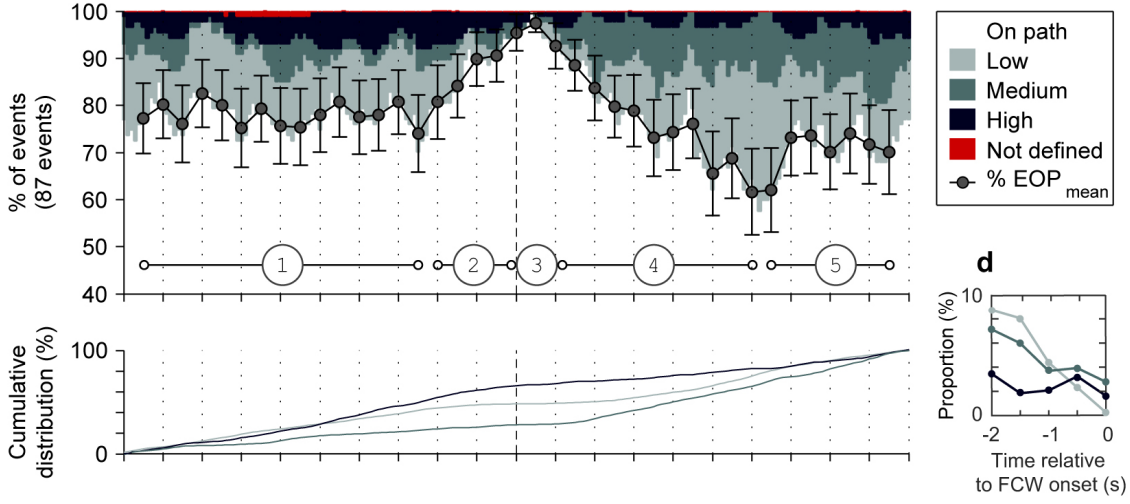

C

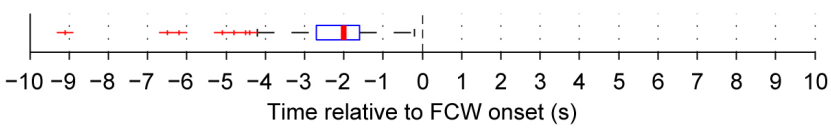

Figure 5. Proportion (a) and cumulative distributions (b) of the glance eccentricity over time, and box plot of the POV (principal other vehicle) brake onset times (c) for the rear-end FCW (frontal collision warning) events. The events are centered at the FCW onset. Numbered circles in (a) indicate the identified intervals described in the text: (1) Steady state driving, (2) Threat anticipation, (3) Threat, (4) Post-threat, (5) (Back to) steady state driving. The curve \%EOPmean shows the mean percentage of eyes on path. The error bars indicate the $95 \%$ confidence interval. In (d) is displayed the proportion of low, medium, and high glance eccentricity over time in the threat anticipation interval.

a

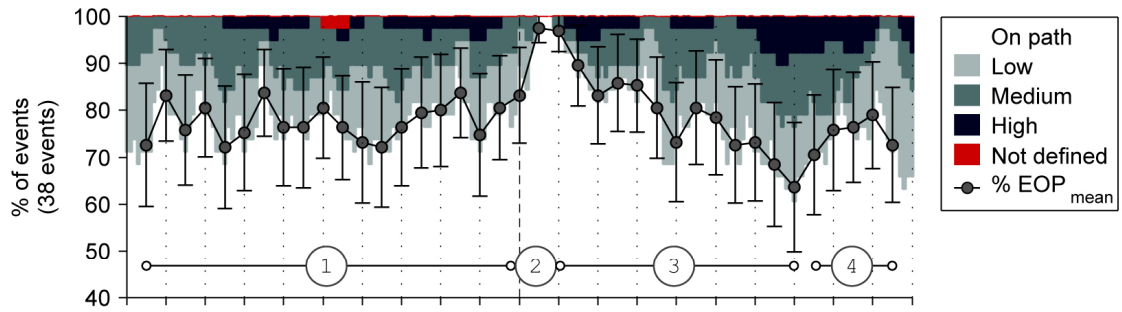

b

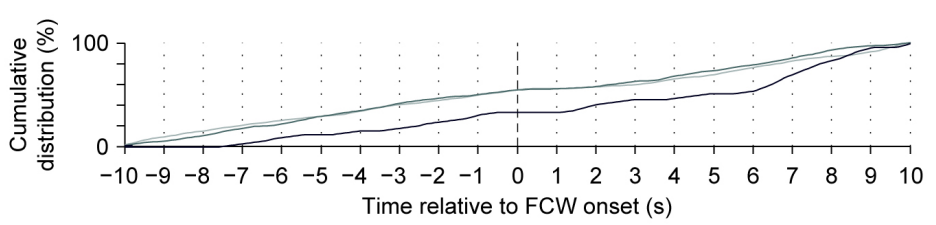

Figure 6. Proportion (a) and cumulative distributions (b) of the glance eccentricity over time for the random FCW (frontal collision warning) events. The events are centered at the FCW onset. Numbered circles in (a) indicate the identified intervals described in the text: (1) Steady state driving, (2) Threat, (3) Postthreat, (4) (Back to) steady state driving. The curve \%EOPmean shows the mean percentage of eyes on path. The error bars indicate the $95 \%$ confidence interval. 


\subsection{Vehicle dynamics}

354

355

356

357

358

359

360

361

362

363

364

365

366

367

368

369

370

371

372

373

374

375

\subsubsection{Rear-end FCW events}

Figure 7 shows the \%EOPmean curve (and the intervals described in section 3.1.1), aligned with the curves of the selected vehicle measures and centered at the FCW onset. In general, the speed and the range to the POV monotonically decreased, with one inflection point after the FCW onset. The range rate had a global minimum of $-5.76 \mathrm{~m} / \mathrm{s}$ at $0.5 \mathrm{~s}$. The longitudinal acceleration had a similar result, but the global minimum of $-3.48 \mathrm{~m} / \mathrm{s}^{2}$ was at $1 \mathrm{~s}$. The $\mathrm{TTC}^{-1}$ and the brake pressure had a global maximum at $1 \mathrm{~s}$, with values of $0.285 \mathrm{~s}^{-1}$ and $29.16 \%$, respectively. The THW behaved differently; it had a global minimum at $1 \mathrm{~s}$ with a value of $1.30 \mathrm{~s}$, and at the end of the post-threat interval there was a noticeable increase in both mean value (4.84 s) and $95 \mathrm{CI}$ as a result of the variable driving conditions. During the (back to) steady state driving interval, THW decreased again. Table 1 reports the values at the interval ends, and the average value in the interval. Note that in the steady state driving interval the vehicle measures were slowly changing over time, as would be expected in car-following, whereas noticeable changes happened between the threat-anticipation and post-threat intervals. At the beginning of the threat-anticipation interval (i.e. at the boundary between intervals 1 and 2 in Figure 7) the value of the longitudinal acceleration was about $-0.20 \mathrm{~m} / \mathrm{s}^{2}$, which is in accordance with the detection threshold found in (Lee et al., 2007). Finally, in the (back to) steady state driving interval, the measures became stable again.

\subsubsection{Random FCW events}

Due to the absence of a POV, the analysis of vehicle measures such as range, range rate, $\mathrm{TTC}^{-1}$, and THW is not relevant in these events. The longitudinal acceleration was negligible and braking was never applied. As a consequence, the speed was steady at around $86 \mathrm{~km} / \mathrm{h}$, a value higher than the one found in section 3.3.1, yet still comparable (Figure 8). 

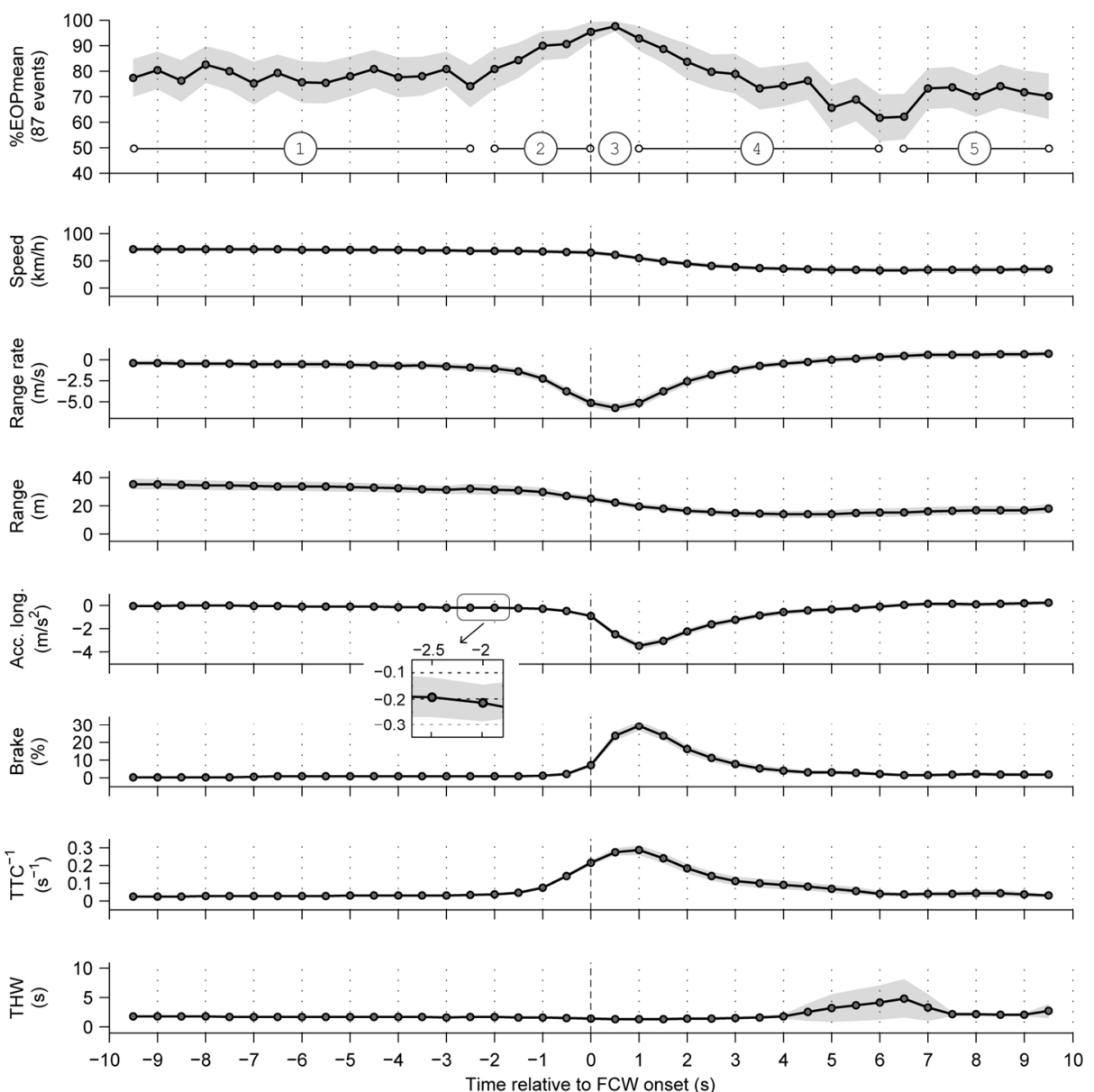

Figure 7. Comparison of \%EOPmean (mean percentage of eyes on path) to several vehicle measures for the rear-end FCW (frontal collision warning) events. The events are centered at the FCW onset. The gray area is the $95 \%$ confidence interval. Numbered circles indicate the identified intervals described in the text: (1) Steady state driving, (2) Threat anticipation, (3) Threat, (4) Post-threat, (5) (Back to) steady state driving.

Table 1. Summary of the values of the vehicle measures for the rear-end FCW (frontal collision warning) events in the identified intervals described in the text. The events are centered at the FCW onset. The values are reported in the format [start, end] (mean).

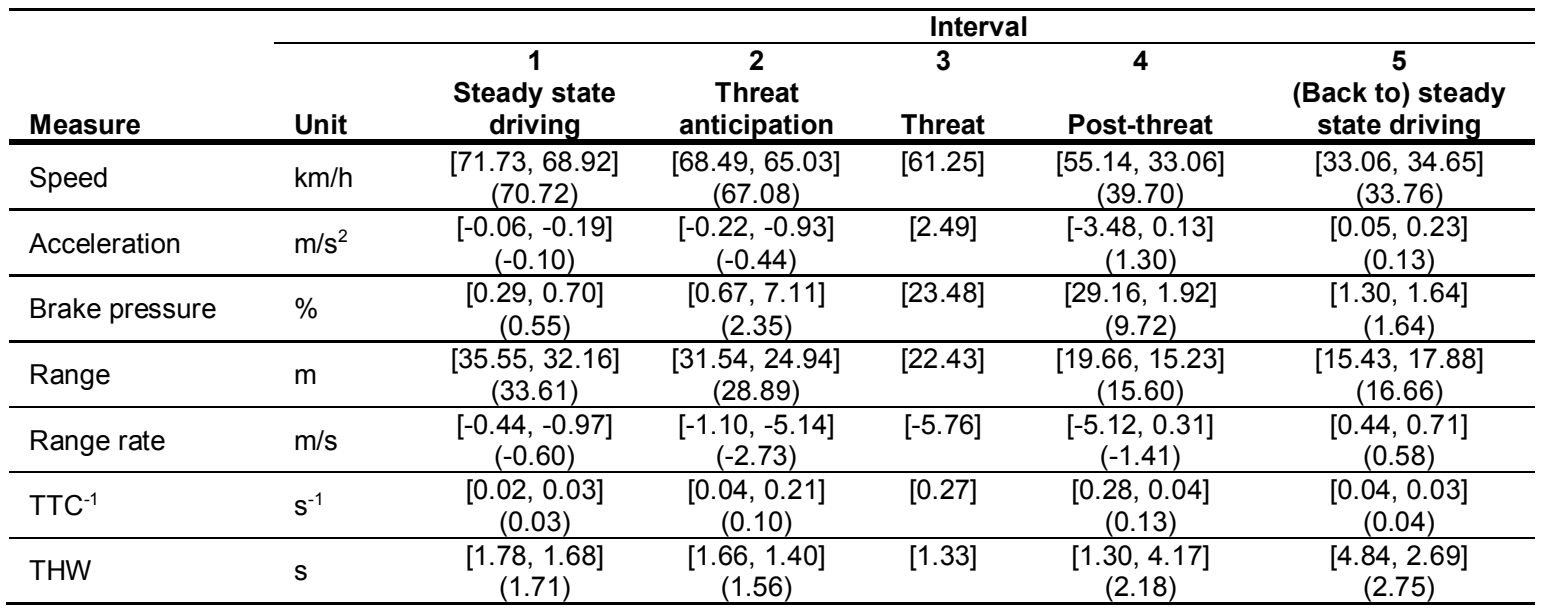



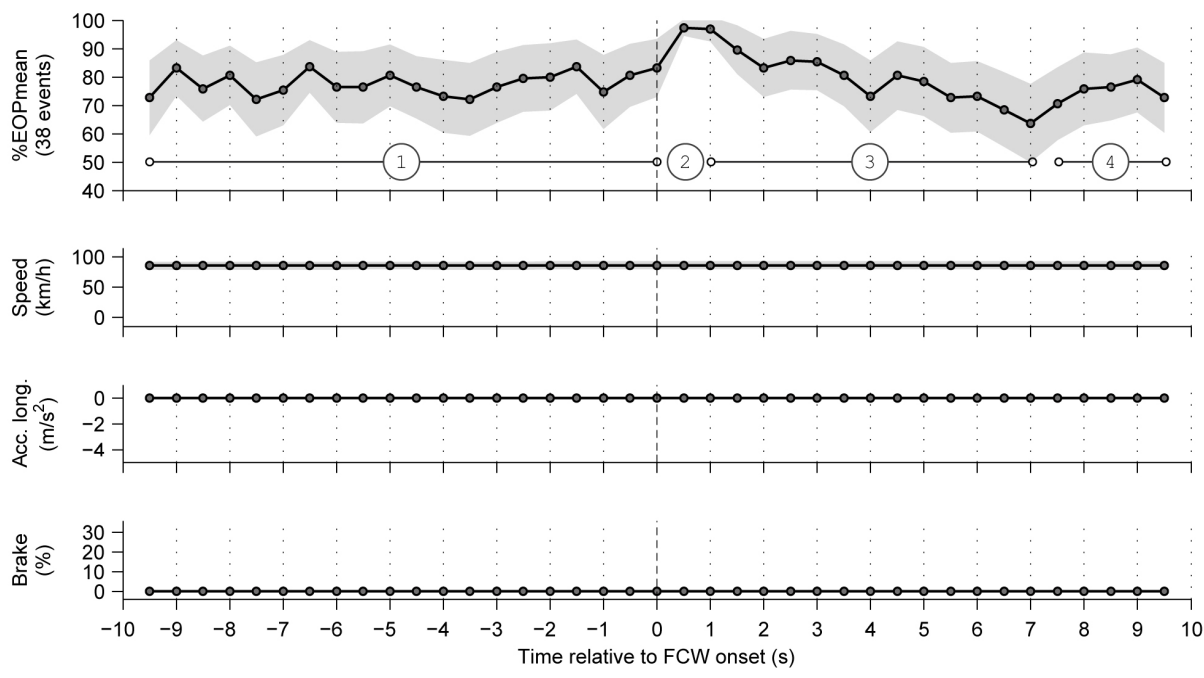

Figure 8. Comparison of \%EOPmean (mean percentage of eyes on path) to the vehicle measures for the random FCW (frontal collision warning) events. The gray area is the $95 \%$ confidence interval. Numbered circles indicate the identified intervals described in the text: (1) Steady state driving, (2) Threat anticipation, (3) Threat, (4) Post-threat, (5) back to steady state driving.

\section{$378 \quad 3.4$ Optical variables}

379 Note that there were no optical variables for the random FCW events due to the absence of a POV. For

380 rear-end FCW events, Figure 9 shows. For the rear-end FCW events, Figure 9 shows the \%EOPmean 381 curve (and the intervals described in section 3.1.1) aligned with the curves of the optical measures associated with the POV, centered at the FCW onset. In general, we can see that $\theta$ steadily increased up to the post-threat interval from 0.062 to $0.202 \mathrm{rad}$, whereas in the (back to) steady state driving interval it slightly decreased. The variables $\dot{\theta}$ and $\tau^{-1}$ had the global maximum at $1 \mathrm{~s}$, with a value of $0.035 \mathrm{rad} / \mathrm{s}$ and $0.284 \mathrm{~s}^{-1}$, respectively.

386 Looking at Table 2, one can note that the variables in the steady state driving interval are slowly 387 changing, and a noticeable change occurs between the threat-anticipation and post-threat intervals. In 388 the threat-anticipation interval, the variable $\dot{\theta}$ crossed the threshold for detecting the looming of the 389 POV when looking on path (of about $0.0036-0.0038 \mathrm{rad} / \mathrm{s}$; see gray area in Table B.1) between $-2 \mathrm{~s}$ and $390-1.5 \mathrm{~s}$. The threshold for detecting the looming at low eccentricity (of about $0.0058-0.0067 \mathrm{rad} / \mathrm{s}$ ) was 391 crossed at about $-1 \mathrm{~s}$. At the end of the events, i.e. in the (back to) steady state driving intervals, the 392 variables began to settle down. 

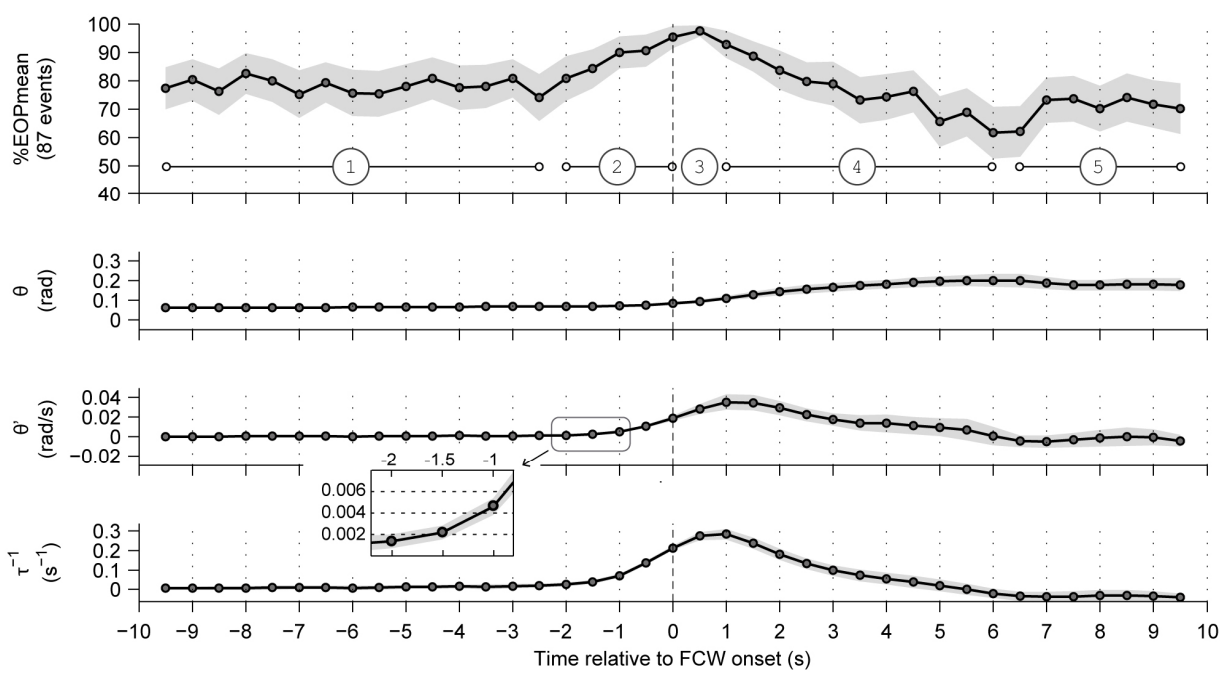

Figure 9. Comparison of \%EOPmean (mean percentage of eyes on path) to the optical measures for the rear-end FCW (frontal collision warning) events. The events are centered at the FCW onset. The gray area is the $95 \%$ confidence interval. Numbered circles indicate the identified intervals described in the text. (1) Steady state driving, (2) Threat anticipation, (3) Threat, (4) Post-threat, (5) (Back to) steady state driving.

Table 2. Summary of the values of the optical variables for the rear-end FCW (frontal collision warning) events in the identified intervals described in the text. The events are centered at the FCW onset. The values are reported in the format [start, end] (mean).

\begin{tabular}{|c|c|c|c|c|c|c|}
\hline \multirow[b]{2}{*}{ Measure } & \multirow[b]{2}{*}{ Unit } & \multicolumn{5}{|c|}{ Interval } \\
\hline & & $\begin{array}{c}1 \\
\text { Steady state } \\
\text { driving }\end{array}$ & $\begin{array}{c}2 \\
\text { Threat } \\
\text { anticipation }\end{array}$ & $\begin{array}{c}3 \\
\text { Threat }\end{array}$ & $\begin{array}{c}4 \\
\text { Post-threat }\end{array}$ & $\begin{array}{c}5 \\
\text { (Back to) steady } \\
\text { state driving }\end{array}$ \\
\hline$\theta$ & $\mathrm{rad}$ & $\begin{array}{c}{[0.062,0.067]} \\
(0.064)\end{array}$ & $\begin{array}{c}0.068,0.083] \\
(0.073)\end{array}$ & [0.094] & $\begin{array}{c}{[0.110,0.202]} \\
(0.168)\end{array}$ & $\begin{array}{c}{[0.201,0.179]} \\
(0.183)\end{array}$ \\
\hline$\dot{\theta}$ & $\mathrm{rad} / \mathrm{s}$ & $\begin{array}{c}{[0.000,0.001]} \\
(0.000)\end{array}$ & $\begin{array}{c}{[0.001,0.019]} \\
(0.007)\end{array}$ & {$[0.028]$} & $\begin{array}{c}{[0.035,0.001]} \\
(0.018)\end{array}$ & $\begin{array}{c}{[-0.005,-0.004]} \\
(-0.003)\end{array}$ \\
\hline$\tau^{-1}$ & $\mathrm{~s}^{-1}$ & $\begin{array}{c}{[0.007,0.022]} \\
(0.012)\end{array}$ & $\begin{array}{c}{[0.027,0.214]} \\
(0.097)\end{array}$ & [0.275] & $\begin{array}{c}{[0.284,-0.020]} \\
(0.101)\end{array}$ & $\begin{array}{c}{[-0.034,-0.041]} \\
(-0.035)\end{array}$ \\
\hline
\end{tabular}

\section{DISCUSSION}

395 This study used data from the naturalistic database EuroFOT (Kessler et al., 2012) to analyze drivers'

396 visual behavior in potential critical situations while driving with ACC. The critical events were selected

397 anytime a FCW (true positive and false positive) was issued while the ACC was active. The visual

398 behavior was examined with respect to two manually-coded metrics, glance location and glance

399 eccentricity, together with other measures based upon several recorded CAN signals. 


\subsection{Visual behavior with respect to the identified intervals}

\subsubsection{Steady state driving interval: glance behavior}

The mean percentage of eyes on path (\%EOPmean) with ACC in the steady state driving interval, in both rear-end and random FCW events, is in accordance with the results in (Tivesten et al., 2015). This study found that the attention to the forward path with ACC was lower than without ( $77 \%$ mean eyes on path with ACC, compared to $\sim 85 \%$ for manual driving). The use of ACC might have reduced the driving task demand, which in turn affected visual attention allocation. While diverting attention away from the forward path could lead to severe consequences, two distinctions must be made: (a) whether the driver's glances off path are driving-related or not, and (b) whether eye behavior is described as 'eyes off path' or 'eyes off threat'. With regard to the former, the results in sections 3.1.1-3.1.2 show that most of the glances off path were driving-related (e.g. towards the driver information module, $\mathrm{L}+\mathrm{R}$ windscreen, rear-view mirror) thus signifying that attention is actually directed towards the driving task. Concerning the latter, discriminating between glances off path and glances off threat is important, taking also into account the benefit of the benefit of the ACC in reducing the risk exposure by maintaining a safe speed and headway, as mentioned in the introduction. The threat anticipation response suggests that drivers direct the eyes off path when there is no impending critical situation, but that they are ready to redirect their visual attention towards a traffic-related threat when needed.

\subsubsection{Threat anticipation interval: what did drivers respond to?}

In accordance with the findings in (Tivesten et al., 2015), Figure 3a shows that drivers anticipated the impending critical situations by directing their eyes to the forward roadway before a situation became critical (that is, before the FCW onset). In line with the literature review presented in the introduction, the results in this study show that there might be three exogenous cues for attracting drivers' attention: deceleration, looming, and brake light cue. The relevance of these cues is corroborated by the analysis of the random FCW events, which demonstrated that the anticipatory response was absent when the cues were absent.

\section{Deceleration cue}

Figure 7 shows that the increase in \%EOPmean in the rear-end FCW events started when the average deceleration of the vehicle in the events was about $0.20 \mathrm{~m} / \mathrm{s}^{2}$, which is in accordance with the detection threshold found in (Lee et al., 2007). This threshold was crossed between $-2.5 \mathrm{~s}$ and $-2 \mathrm{~s}$, at the beginning of the threat-anticipation interval. This mild deceleration might have been the effect of either the throttle release or the brake onset by the ACC. This cue might have informed the drivers of the approaching phase to a POV, making them look at the road. This behavior seems to be in line with the subjective data collected by Fancher et al. (1998), which indicates that drivers perceived the ACC-induced deceleration as a cue to look ahead because of an arising headway conflict. 
435 Figure 5a shows that the steady decrease of glances towards low eccentricity locations was the main 436 contributor to the steady increase in \%EOPmean in the rear-end FCW events. The glances at medium 437 and, in particular, at high eccentricity locations were less influenced, up until the onset of the FCW. 438 When using the detection threshold for the optical variable $\dot{\theta}$ (because, unlike $\tau^{-1}$, this variable is less sensitive to the different experimental setup, as shown in Appendix B), the threshold for detecting the closure of the POV at low eccentricity was approximately at $-1 \mathrm{~s}$, which is $1 \mathrm{~s}$ later than the onset of the deceleration cue. This finding suggests that the deceleration cue is more effective than the looming stimulus (in the drivers' peripheral vision) at redirecting visual attention towards the forward path, since the former is detected sooner. However, once the glance is on the forward roadway, drivers might rely on the looming stimulus to perceive the imminent threat. Thus the driver keeps looking at the road, without diverting attention away, when the looming is detectable. In other words, the looming threshold value was initially crossed when looking at the forward path at the beginning of the threat anticipation interval (i.e., between $-2 \mathrm{~s}$ and $-1.5 \mathrm{~s}$ ); thereafter the glance continued to be on path (for example, as an effect of the deceleration cue) because the looming of the lead vehicle was above threshold. Note that the looming threshold reference values summarized in the introduction and in Table B.1 were gathered from studies that were carried out with alert drivers in favorable conditions on an empty road (Lamble et al., 1999; Summala et al., 1998). In contrast, looming detection may be more difficult in real-world driving, due to cluttered driving scenes (e.g. in congested traffic) and less favorable weather and lighting conditions (Victor et al., 2015).

\section{Brake light cue}

455 Figure $5 \mathrm{c}$ shows that the beginning of the steady state driving interval corresponded to the median of 456 the distribution of the POV brake light onset times across the events. This correspondence suggests that 457 brake light onset may be influencing drivers to look at the road. However, as pointed out in the 458 introduction, previous studies have yielded conflicting results. It is difficult to assess from our results 459 whether the brake lights increase the drivers' visual attention to the forward path. The majority of events 460 in this study ( $87 \%$ ) occurred in daylight and clear weather (see Table A.3), which reduced the saliency 461 of the brake light stimulus; Summala et al. (1998) found that detection was significantly impaired in the 462 visual periphery, even at a low level of eccentricity. This suggests that the brake lights might not be 463 intense enough to capture the driver's attention in daylight and clear weather circumstances when the 464 driver isn't looking directly ahead. However, in adverse conditions and for night driving, the brake lights 465 might be more easily detected at the periphery and they could have played a primary role in the detection 466 of lead vehicle looming (Janssen, 1974). However, note that even if the brake light stimulus is salient 467 enough it still would not necessarily be the cue that causes a brake reaction or inhibits an off-road glance, as argued by Victor et al. (2015) and Markkula et al. (2016). 


\subsubsection{Threat interval: how did drivers respond?}

470 Figure 5a shows that the onset of the FCW redirected the glances at medium and, in particular, at high eccentricity locations. However, as a consequence of the threat anticipation, the \%EOPmean was already $95.4 \%$ at the onset of the FCW. Interestingly, the random FCW events clearly showed a rapid orienting effect of the FCW (Figure 4 and Figure 6). These results suggest that the FCW was effective at making the drivers glance at the forward path, but the absence of a threat allowed drivers to quickly divert their attention again.

\subsubsection{Post-threat interval: what did drivers do after the response?}

Figure 3 shows that drivers started to divert visual attention away from the forward path just after the onset of the FCW. The increase of glances towards the rear-view mirror was particularly noticeable from $1 \mathrm{~s}$ after the FCW onset. This behavior might be interpreted as an improvement of situation awareness (e.g. checking if the driver vehicle was about to be struck by a following one). In the end of the postthreat interval one can notice a large proportion of glances towards the driver information module. This behavior might be the consequence of the drivers' controlling the ACC settings or re-engaging the system. Although less noticeable, the post-threat interval was also found in the random FCW (Figure 4). It could be argued that the increased frequency of glances to the driver information module and other types of glances indicate enhanced situation awareness: for example, drivers may look towards the driver information module to search the settings for the cause of the warning, and towards other areas to check for the presence of objects in front of the car.

\subsection{Outstanding question}

Why did the drivers receive a FCW even when they were already looking at the forward path and anticipated the threat? Here we propose to answer this question in terms of trust, expectancy, and satisficing behavior.

492 Trust

493 Trust can be defined as "the attitude that an agent will help achieve an individual's goals in a situation characterized by uncertainty and vulnerability" (Lee \& See, 2004, p. 54). In this regard, the driver would have waited until the last second, i.e. the FCW onset, before deciding to intervene and override the ACC,

496 for example by manually braking, as suggested in (Rudin-Brown \& Parker, 2004). Larsson et al. (2014)

497 argue that an increased brake reaction time during ACC control is not necessarily a disadvantage of the 498 system; experienced drivers clearly trust the ACC to perform its task appropriately, so they only 499 intervene at the last second.

\section{Expectancy}

501 Expectancy is the subjective prediction, associated with a degree of uncertainty, about how a specific 502 situation will develop, based on previous experience and contextual information (Engström et al., 2013; 503 Sanders, 1966). Expectancy largely regulates the driver's behavior in steady state driving (Engström et 
al., 2013). If the need for a response is expected to disappear, drivers might delay their response

505 (Summala, 2000). For example, the drivers could have waited to proactively brake because they did not

506 expect the slowing leading car to suddenly brake, or they expected it to start accelerating again. When

507 the FCW was issued and the drivers detected that the situation didn't develop as expected, they acted in

508 order to recover the safety margins.

\section{Satisficing behavior}

510 Satisficing behavior is the behavior supported by the notion of acceptable, rather than optimal, 511 performance (Boer, 1999). In normal driving drivers tend to satisfice to remain within a subjective 512 comfort zone whose boundaries are primarily determined by safety margins (Summala, 2007). 513 Additionally, the comfort zone's boundary may be stretched by extra motives (Summala, 2007), from 514 which the driver could gain a benefit that justifies the cost of getting closer to the discomfort zone. For 515 example, drivers may decide to wait until the last moment before pressing the brake pedal, thus 516 decreasing the following distance (cost), in order to avoid disengaging, and having to reengage, the ACC 517 (benefit).

\section{$518 \quad 4.3$ Limitations}

519 Naturalistic studies have some limitations intrinsic to their design, such as lack of experimental control of participants, scenarios, and vehicle systems. These same considerations should be taken into account in interpreting these results. Video data reduction (such as eye glance behavior) was conducted by the primary author. Inter-rater reliability was not established.

\section{CONCLUSION}

This study corroborates and extends the results from (Tivesten et al., 2015) and proposes an explanation for drivers' reactions to potential critical situations, identified as FCW onsets while ACC is active. The findings indicate that vestibular/somatosensory and visual cues (i.e., deceleration, looming, and lead vehicle brake lights) attract the drivers' attention to the forward road before the onset of the FCW. This explanation is further supported by the absence of an anticipatory response in the random FCW events, in which these three cues were absent.

530 It is argued that the deceleration cue might be the predominant cue for triggering glances towards the forward roadway before a longitudinal threat develops into a conflict. (If this argument is correct, simulator experiments could, whenever possible, exploit moving base motion cues to re-engage the drivers when an intervention is required in critical situations.) Once the driver's attention has been captured, the looming cue (together with the brake light cue) is probably the main stimulus maintaining the driver attention to the forward path, providing more information about an impending conflict and supporting the driver's response. 
The findings provide evidence of two kind of driver responses, to a warning and to a threat. The response to a warning is characterized by a quick (but temporary) reorientation to the forward path, whereas the response to a threat is characterized by a slower, longer-lasting increase of glances on-path. The former behavior is particularly noticeable in the random FCW events.

The random FCW events clearly show that, when there is a warning without an external threat (and visual and deceleration cues are not provided), there is no anticipatory response. These events show that the FCW alone acts as an effective attention-orienting mechanism. In contrast, in rear-end FCW events, the FCW was effective at re-orienting the glances that were further away from the forward roadway (i.e., at medium and high levels of eccentricity).

This study also showed that the time-course of visual behavior in critical situations can be divided into intervals with different glance characteristics according to the driving situation. The identified intervals were defined as steady state driving, threat-anticipation, threat, post-threat and (back to) steady state driving. These contextually defined intervals are essential for understanding the attention response process.

This work is also of interest for automated driving research, because it provides additional information about drivers' perception of the driving situation and shows how important visual and vestibular/somatosensory cues can be for alerting drivers to critical situations, and for starting planning a proper avoidance action. Furthermore, these results are relevant for the design of higher levels of automation, specifically when re-engaging the driver if the system automation capabilities are exceeded. The results from this study suggest that it is important to design a vehicle that can communicate system limitations through actuation and to develop a warning strategy that incorporates knowledge of driver glance responses to safety systems.

\section{ACKNOWLEDGEMENTS}

This research was financially supported by the European Marie Curie ITN project HFAuto (Human Factors of Automated driving, PITN-GA-2013-605817). The study was performed at SAFER, the vehicle and traffic safety center at Chalmers University of Technology (Gothenburg, Sweden). The authors would like to thank John D. Lee for his feedback and inspiring conversations, the colleagues at the Accident Prevention group at SAFER for their comments and suggestions on this paper, and Kristina Mayberry for language review and editing.

\section{REFERENCES}

Boer, E. R. (1999). Car following from the driver's perspective. Transportation Research Part F: Traffic Psychology and Behaviour, 2(4), 201-206.

Coelingh, E., Jakobsson, L., Lind, H., \& Lindman, M. (2007). Collision warning with auto brake: a real-life safety perspective. 
de Winter, J. C. F., Happee, R., Martens, M. H., \& Stanton, N. A. (2014). Effects of adaptive cruise control and highly automated driving on workload and situation awareness: A review of the empirical evidence. Transportation Research Part F: Traffic Psychology and Behaviour, 27, 196-217. doi:10.1016/j.trf.2014.06.016

Dozza, M., Moeschlin, F., \& Léon-Cano, J. (2010). FOTware: A modular, customizable software for analysis of multiple-source field-operational-test data. Paper presented at the Second international symposium on naturalistic driving research.

Endsley, M. R. (1988, 23-27 May 1988). Situation awareness global assessment technique (SAGAT). Paper presented at the Aerospace and Electronics Conference, 1988. NAECON 1988., Proceedings of the IEEE 1988 National.

Engström, J., Victor, T., \& Markkula, G. (2013). Attention selection and multitasking in everyday driving: A conceptual model. In M. T. W. Victor, J. D. Lee, \& M. A. Regan (Eds.), Driver Distraction and Inattention: Advances in Research and Countermeasures: Ashgate Publishing, Ltd.

Fancher, P., Ervin, R., Sayer, J., Hagan, M., Bogard, S., Bareket, Z., . . . Haugen, J. (1998). Intelligent cruise control field operational test. Final report (DOT HS 808 849). Retrieved from http://www.nhtsa.gov/DOT/NHTSA/NRD/Multimedia/PDFs/Crash\%20Avoidance/1998/icc1998.pdf

Franconeri, S., \& Simons, D. (2003). Moving and looming stimuli capture attention. Perception \& Psychophysics, 65(7), 999-1010. doi:10.3758/BF03194829

Hoffmann, E. R. (1968). Detection of vehicle velocity changes in car following. Paper presented at the Australian Road Research Board Conference Proc.

Hoffmann, E. R., \& Mortimer, R. G. (1994a). Drivers' estimates of time to collision. Accident Analysis \& Prevention, 26(4), 511-520.

Hoffmann, E. R., \& Mortimer, R. G. (1994b). Scaling of relative velocity between vehicles. Paper presented at the Proceedings of the Human Factors and Ergonomics Society Annual Meeting.

Janssen, W. H. (1974). The perception of manoeuvres of moving vehicles. Progress report VI (final report): Implications of psychophysical threshold measurements for the night driving situation. Retrieved from

Jonides, J. (1981). Voluntary versus automatic control over the mind's eye's movement. Attention and performance $I X, 9,187-203$.

Kessler, C., Etemad, A., Alessandretti, G., Heinig, K., Brouwer, R., Cserpinszky, A., . . Benmimoun, M. (2012). EuroFOT Deliverable D11. 3 - Final Report. Retrieved from http://www.eurofot-ip.eu/

Klauer, S. G., Dingus, T. A., Neale, V. L., Sudweeks, J. D., \& Ramsey, D. J. (2006). The impact of driver inattention on near-crash/crash risk: An analysis using the 100-car naturalistic driving study data. Retrieved from https://vtechworks.lib.vt.edu/bitstream/handle/10919/55090/DriverInattention.pdf?sequence=1

Klein, R., Kingstone, A., \& Pontefract, A. (1992). Orienting of Visual Attention. In K. Rayner (Ed.), Eye Movements and Visual Cognition (pp. 46-65): Springer New York.

Lamble, D., Laakso, M., \& Summala, H. (1999). Detection thresholds in car following situations and peripheral vision: Implications for positioning of visually demanding in-car displays. Ergonomics, 42(6), 807-815.

Lee, D. N. (1976). A theory of visual control of braking based on information about time-to-collision. Perception, 5(4), 437-459. doi:10.1068/p050437

Lee, J. D., McGehee, D., Brown, T., \& Marshall, D. (2006). Effects of adaptive cruise control and alert modality on driver performance. Transportation Research Record: Journal of the Transportation Research Board(1980), 49-56.

Lee, J. D., McGehee, D. V., Brown, T. L., \& Nakamoto, J. (2007). Driver sensitivity to brake pulse duration and magnitude. Ergonomics, 50(6), 828-836. doi:10.1080/00140130701223220

Lee, J. D., \& See, K. A. (2004). Trust in automation: designing for appropriate reliance. Human Factors, 46(1), 50-80. doi:10.1518/hfes.46.1.50 30392

Lin, J. Y., Franconeri, S., \& Enns, J. T. (2008). Objects on a Collision Path With the Observer Demand Attention. Psychological science, 19(7), 686-692. doi:10.1111/j.1467-9280.2008.02143.x

Malta, L., Aust, M. L., Faber, F., Metz, B., Pierre, G. S., Benmimoun, M., \& Schäfer, R. (2011). EuroFOT Deliverable 6.4 - Final results: Impacts on traffic safety. Retrieved from http://www.eurofot-ip.eu/

Markkula, G., Engström, J., Lodin, J., Bärgman, J., \& Victor, T. (2016). A farewell to brake reaction times? Kinematics-dependent brake response in naturalistic rear-end emergencies (Manuscript submitted for publication).

Michon, J. A. (1985). A critical view of driver behavior models: what do we know, what should we do? Human behavior and traffic safety (pp. 485-524): Springer.

Mortimer, R. G. (1990). Perceptual factors in rear-end crashes. Paper presented at the Proceedings of the Human Factors and Ergonomics Society Annual Meeting.

NHTSA. (2005). Automotive Collision Avoidance System Field Operational Test (ACAS FOT) - Final Program Report.

Retrieved from 

0Final\%20Program\%20Report\%20DOT\%20HS\%20809\%20886.pdf

NHTSA. (2015). US Department of Transportation Releases Policy on Automated Vehicle Development. Retrieved from

Nilsson, J., Strand, N., Falcone, P., \& Vinter, J. (2013, 24-27 June 2013). Driver performance in the presence of adaptive cruise control related failures: Implications for safety analysis and fault tolerance. Paper presented at the Dependable Systems and Networks Workshop (DSN-W), 2013 43rd Annual IEEE/IFIP Conference on. OECD. (1990). Behavioural adaptations to changes in the road transport system (9264133895). Retrieved from

Ranney, T. A. (1994). Models of driving behavior: a review of their evolution. Accident Analysis \& Prevention, 26(6), 733-750.

Regan, D., \& Vincent, A. (1995). Visual processing of looming and time to contact throughout the visual field. Vision Research, 35(13), 1845-1857.

Rudin-Brown, C. M., \& Parker, H. A. (2004). Behavioural adaptation to adaptive cruise control (ACC): implications for preventive strategies. Transportation Research Part F: Traffic Psychology and Behaviour, 7(2), 59-76. doi:10.1016/j.trf.2004.02.001

SAE. (2014). J3016 - Taxonomy and definitions for terms related to on-road motor vehicle automated driving systems: SAE International.

Sanders, A. F. (1966). Expectancy: Application and measurement. Acta Psychologica, 25, 293-313. doi:http://dx.doi.org/10.1016/0001-6918(66)90013-8

Shinar, D. (2007). Vision, Visual Attention, and Visual Search Traffic Safety and Human Behavior (pp. 91-123): Emerald, Inc.

Stanton, N. A., Young, M., \& McCaulder, B. (1997). Drive-by-wire: the case of driver workload and reclaiming control with adaptive cruise control. Safety Science, 27(2), 149-159.

Strand, N., Nilsson, J., Karlsson, I. M., \& Nilsson, L. (2014). Semi-automated versus highly automated driving in critical situations caused by automation failures. Transportation Research Part F: Traffic Psychology and Behaviour, 27, 218-228.

Summala, H. (2000). Brake Reaction Times and Driver Behavior Analysis. Transportation Human Factors, 2(3), 217-226. doi:10.1207/sthf0203_2

Summala, H. (2007). Towards understanding motivational and emotional factors in driver behaviour: comfort through satisficing Modelling driver behaviour in automotive environments (pp. 189-207): Springer.

Summala, H., Lamble, D., \& Laakso, M. (1998). Driving experience and perception of the lead car's braking when looking at in-car targets. Accident Analysis \& Prevention, 30(4), 401-407. doi:10.1016/s0001-4575(98)000050

Tivesten, E., Morando, A., \& Victor, T. (2015). The timecourse of driver visual attention in naturalistic driving with Adaptive Cruise Control and Forward Collision Warning. Paper presented at the 4th International Driver Distraction and Inattention Conference, Sydney, New South Wales.

Victor, T., Dozza, M., Bärgman, J., Boda, C.-N., Engström, J., Flannagan, C., . . Markkula, G. (2015). SHRP2 Analysis of naturalistic driving study data: Safer glances, driver inattention and crash risk. Retrieved from http://www.trb.org/Publications/PubsSHRP2ResearchReportsSafety.aspx

Victor, T. W., Engström, J., \& Harbluk, J. L. (2008). Distraction Assessment Methods Based on Visual Behavior and Event Detection Driver Distraction (pp. 135-165): CRC Press.

Wege, C., Will, S., \& Victor, T. (2013). Eye movement and brake reactions to real world brake-capacity forward collision warnings--a naturalistic driving study. Accident Analysis \& Prevention, 58, 259-270. doi:10.1016/j.aap.2012.09.013

Young, M. S., \& Stanton, N. A. (2007). Back to the future: brake reaction times for manual and automated vehicles. Ergonomics, 50(1), 46-58. doi:10.1080/00140130600980789 
Table A.1 Coding scheme for the glance location. The coding was adapted from (Victor et al., 2015, p. 24)

\begin{tabular}{|c|c|c|}
\hline $\begin{array}{l}\text { Color } \\
\text { code }\end{array}$ & Glance location* & Description \\
\hline $\mathrm{O}$ & On path & $\begin{array}{l}\text { Any glance directed toward the direction of the vehicle's travel. When the } \\
\text { vehicle is turning, these glances may be directed toward the vehicle's heading. }\end{array}$ \\
\hline O & Center stack & Any glance to the vehicle's vertical center stack. \\
\hline O & $\begin{array}{l}\text { Driver information } \\
\text { module }\end{array}$ & $\begin{array}{l}\text { Any glance to the driver information module (e.g., speedometer, control stalks, } \\
\text { and steering wheel). }\end{array}$ \\
\hline O & Phone & $\begin{array}{l}\text { Any glance at a cell phone or other electronic communications device, no } \\
\text { matter where it is located. }\end{array}$ \\
\hline O & Interior object & $\begin{array}{l}\text { Any glance to an identifiable object in the vehicle (e.g., personal items, the } \\
\text { cup-holder area between passenger seat and driver seat) }\end{array}$ \\
\hline O & Passenger & $\begin{array}{l}\text { Any glance to a passenger, whether in front or rear seat. Context will be } \\
\text { needed (e.g., they're talking or passing something) in some situations. }\end{array}$ \\
\hline O & Eyes closed & $\begin{array}{l}\text { Any time that both participant's eyes are closed outside of normal blinking } \\
\text { (e.g., the subject is falling asleep or rubbing eyes). }\end{array}$ \\
\hline ○ & Other & $\begin{array}{l}\text { Any glance that cannot be categorized using the above codes (e.g., the driver } \\
\text { looks straight up at the sky as if watching a plane fly by, the driver looks at the } \\
\text { bonnet, the driver is tilting his or her head back to drink and the eyes leave the } \\
\text { forward glance but do not really focus on anything at all...). }\end{array}$ \\
\hline 0 & Rear-view mirror & Any glance to the rearview mirror. \\
\hline $\mathrm{O}$ & $\begin{array}{l}\mathrm{L}+\mathrm{R} \text { Side mirror } \\
\mathrm{L}+\mathrm{R} \text { Window }\end{array}$ & Any glance to the left or right side mirror or window. \\
\hline O & $L+R$ windscreen & $\begin{array}{l}\text { Any glance out the forward windshield when the driver appears to be looking } \\
\text { out the windshield but clearly not in the direction of travel (e.g., at road signs or } \\
\text { buildings) }\end{array}$ \\
\hline O & L+R over shoulder & $\begin{array}{l}\text { Any glance over either of the participant's shoulders. In general, this will } \\
\text { require the eyes to pass the B-pillar. If over the left shoulder, the eyes may not } \\
\text { be visible, but this glance location can be inferred from context. }\end{array}$ \\
\hline O & No eyes visible & $\begin{array}{l}\text { Glance location unknown: Unable to complete glance analysis due to an } \\
\text { inability to see the driver's eyes/face (due to obstruction or glare). }\end{array}$ \\
\hline $\mathbf{0}$ & No video & $\begin{array}{l}\text { Unable to complete glance analysis because the video source is temporarily } \\
\text { unavailable. }\end{array}$ \\
\hline O & Not coded & Time-series data for which glance annotation was not performed. \\
\hline
\end{tabular}

Table A.2 Coding scheme for the glance eccentricity.

\begin{tabular}{|c|c|c|c|}
\hline $\begin{array}{l}\text { Color } \\
\text { code }\end{array}$ & Eccentricity level & Average angle & Area of interest \\
\hline 0 & On path & Between $0^{\circ}$ and $10^{\circ}$ & On path \\
\hline 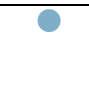 & Low & Between $10^{\circ}$ and $30^{\circ}$ & $\begin{array}{l}\mathrm{L} \text { windscreen } \\
\mathrm{R} \text { windscreen } \\
\text { Driver information module }\end{array}$ \\
\hline 0 & Medium & Between $30^{\circ}$ and $60^{\circ}$ & $\begin{array}{l}\text { L side mirror } \\
\text { L window } \\
\text { Rear-view mirror } \\
\text { Center stack }\end{array}$ \\
\hline 0 & High & Above $60^{\circ}$ & $\begin{array}{l}\text { R side mirror } \\
\text { R window } \\
\text { L over shoulder } \\
\text { R over shoulder } \\
\text { Interior object } \\
\text { Passenger } \\
\text { Phone } \\
\text { Other } \\
\text { Eyes closed }\end{array}$ \\
\hline 0 & Not defined & Not defined & $\begin{array}{l}\text { Eyes not visible } \\
\text { No video } \\
\text { Not coded }\end{array}$ \\
\hline
\end{tabular}


Table A.3 Summary of the annotations of the environmental variables.

\begin{tabular}{|c|c|c|c|}
\hline \multirow[b]{2}{*}{ Variable } & \multirow[b]{2}{*}{ Category } & \multicolumn{2}{|c|}{ Number of events } \\
\hline & & $\begin{array}{c}\text { Rear-end FCW } \\
\text { (total of } 87 \text { events) }\end{array}$ & $\begin{array}{c}\text { Random FCW } \\
\text { (total of } 38 \text { events) }\end{array}$ \\
\hline \multirow[t]{4}{*}{ Lightening } & Daylight & 71 & 12 \\
\hline & Darkness lighted & 12 & 8 \\
\hline & Darkness unlighted & 2 & 8 \\
\hline & Artificial darkness (e.g. tunnel) & 2 & 10 \\
\hline \multirow[t]{3}{*}{ Weather condition } & No adverse condition & 80 & 37 \\
\hline & Mist/Light rain & 3 & 1 \\
\hline & Rain & 4 & 0 \\
\hline \multirow[t]{2}{*}{ Road geometry } & Straight road & 80 & 31 \\
\hline & Curve & 7 & 7 \\
\hline
\end{tabular}




\section{APPENDIX B. COMPILATION OF RELATED STUDIES ON THE LOOMING DETECTION THRESHOLD}

Table B.1 presents a compilation of related research on the threshold for detecting the looming of a lead

687 vehicle as a function of glance eccentricity. Summala et al. (1998) and Lamble et al. (1999) performed 688 test track studies in which the participants were asked to detect the lead vehicle closure while performing 689 a visual secondary task. The visual secondary task forced the participants to look away from the forward 690 pathway at increasing degrees of eccentricity. The methodologies in the two papers were similar except 691 for (a) different experimental conditions (lead vehicle deceleration, following speed, range and degrees 692 of eccentricity) and (b) different measures presented in the results. Summala et al. (1998) provided the 693 detection threshold in terms of $\tau^{-1}$, whereas Lamble et al. (1999) provided the results in terms of $\dot{\theta}$ 694 and TTC. To facilitate the comparison, we integrated and merged the results.

695 The results in (Summala et al., 1998) were integrated by computing the detection threshold in terms of

$696 \dot{\theta}$. The car-following scenario used in the study was simulated by implementing a MATLAB-based 697 script, and the values of $\dot{\theta}$ at detection were calculated via the equations in section 2.5. (Please note 698 that only the data at $60 \mathrm{~km} / \mathrm{h}$ were used since the ACC in the present study was not active at speeds 699 below $30 \mathrm{~km} / \mathrm{h}$.) The results in (Lamble et al., 1999) were integrated by approximating TTC with $\tau^{-1}$ 700 (Lee, 1976). (Please note that the data at $4^{\circ}$ were excluded as suggested by the authors in (Lamble et al., 701 1999).)

702 Subsequently, the looming detection thresholds at eccentricities of $0^{\circ}, 30^{\circ}, 60^{\circ}$ and $90^{\circ}$ were predicted 703 via linear regression. Moreover, the results obtained under varying experimental conditions (i.e., range and following speed) were merged, to obtain an average reference value for $\dot{\theta}$ and $\tau^{-1}$ (gray area in Table B.1). 
Table B.1 Compilation of related results from test track studies on the visual perceptual threshold in terms of $\dot{\theta}$ and $\tau^{-1}$. The detection threshold, as a function of glance eccentricity, was predicted via regression at $0^{\circ}, 30^{\circ}, 60^{\circ}$, and $90^{\circ}$. The gray area indicates the analysis over the merged data provided by the studies.

\begin{tabular}{|c|c|c|c|c|}
\hline Study & Variable & $\begin{array}{l}\text { Predict } \\
\text { functiol }\end{array}$ & $\begin{array}{l}\text { shold as a } \\
\text { ance eccentricity }\end{array}$ & Notes \\
\hline \multirow[t]{28}{*}{$\begin{array}{l}\text { Summala et al. } \\
(1998)\end{array}$} & & & & $\begin{array}{l}\text { Test track study. Lead vehicle }(1.62 \mathrm{~m} \text { wide) } \\
\text { braking at } \sim-2.1 \mathrm{~m} / \mathrm{s}^{2} \text { with brake lights } \\
\text { deactivated. Clear weather. Participants were } \\
\text { requested to brake as soon as they noticed } \\
\text { the lead vehicle approaching. }\end{array}$ \\
\hline & & & & Range $=30 \mathrm{~m}, \mathrm{speed}=60 \mathrm{~km} / \mathrm{h}$ \\
\hline & & 0.0051 & $@ 0^{\circ}$ & \\
\hline & $\dot{\theta}$ & 0.0073 & $@ 30^{\circ}$ & $\dot{\theta}=0.0050781+0.00007445 \cdot x^{\circ}$ \\
\hline & {$[\mathrm{rad} / \mathrm{s}]$} & 0.0095 & $@ 60^{\circ}$ & $\mathrm{R}^{2}=0.94$ \\
\hline & & 0.0118 & $@ 90^{\circ}$ & \\
\hline & & 0.0939 & $@ 0^{\circ}$ & \\
\hline & $\tau^{-1}$ & 0.1348 & $@ 30^{\circ}$ & $\tau^{-1}=0.093899+0.0013646 \cdot x^{\circ}$ \\
\hline & {$\left[\mathrm{s}^{-1}\right]$} & 0.1758 & $@ 60^{\circ}$ & $R^{2}=0.93$ \\
\hline & & 0.2167 & $@ 90^{\circ}$ & \\
\hline & & & & Range $=60 \mathrm{~m}$, speed $=60 \mathrm{~km} / \mathrm{h}$ \\
\hline & & 0.0022 & $@ 0^{\circ}$ & \\
\hline & $\dot{\theta}$ & 0.0043 & $@ 30^{\circ}$ & $\dot{\theta}=0.0022014+0.000069697 \cdot x^{\circ}$ \\
\hline & $\theta$ & 0.0064 & $@ 60^{\circ}$ & $\mathrm{R}^{2}=0.94$ \\
\hline & & 0.0085 & $@ 90^{\circ}$ & \\
\hline & & 0.0815 & $@ 0^{\circ}$ & \\
\hline & $\tau^{-1}$ & 0.1569 & $@ 30^{\circ}$ & $\tau^{-1}=0.081462+0.0025156 \cdot x^{\circ}$ \\
\hline & & 0.2324 & $@ 60^{\circ}$ & $\mathrm{R}^{2}=0.93$ \\
\hline & & 0.3079 & $@ 90^{\circ}$ & \\
\hline & & & & Range $=30-60 \mathrm{~m}$, speed $=60 \mathrm{~km} / \mathrm{h}$ \\
\hline & & 0.0036 & $@ 0^{\circ}$ & \\
\hline & $\dot{\theta}$ & 0.0058 & $@ 30^{\circ}$ & $\dot{\theta}=0.0036397+0.000072073 \cdot x^{\circ}$ \\
\hline & $\theta$ & 0.0080 & $@ 60^{\circ}$ & $\mathrm{R}^{2}=0.42$ \\
\hline & & 0.0101 & $@ 90^{\circ}$ & \\
\hline & & 0.0877 & $@ 0^{\circ}$ & \\
\hline & $\tau^{-1}$ & 0.1459 & $@ 30^{\circ}$ & $\tau^{-1}=0.08768+0.0019401 \cdot x^{\circ}$ \\
\hline & $\tau$ & 0.2041 & $@ 60^{\circ}$ & (l) \\
\hline & & 0.2623 & $@ 90^{\circ}$ & \\
\hline \multirow[t]{28}{*}{$\begin{array}{l}\text { Lamble et al. } \\
\text { (1999) }\end{array}$} & & & & $\begin{array}{l}\text { Test track study. Lead vehicle }(1.62 \mathrm{~m} \text { wide }) \\
\text { coasting at } \sim-0.7 \mathrm{~m} / \mathrm{s}^{2} \text {. Clear weather. } \\
\text { Participants were requested to brake as soon } \\
\text { as they noticed the lead vehicle approaching. }\end{array}$ \\
\hline & & & & Range $=20 \mathrm{~m}$, speed $=50 \mathrm{~km} / \mathrm{h}$ \\
\hline & & 0.0038 & $@ 0^{\circ}$ & \\
\hline & $\dot{\theta}$ & 0.0072 & $@ 30^{\circ}$ & $\dot{\theta}=0.0038459+0.0001116 \cdot x^{\circ}$ \\
\hline & $\theta$ & 0.0105 & $@ 60^{\circ}$ & $\mathrm{R}^{2}=0.85$ \\
\hline & & 0.0139 & $@ 90^{\circ}$ & \\
\hline & & 0.2357 & $@ 0^{\circ}$ & \\
\hline & $\tau_{-1}^{-1}$ & 0.2569 & $@ 30^{\circ}$ & $\tau^{-1}=0.23567+0.00070727 \cdot x^{\circ}$ \\
\hline & $\tau^{-1}$ & 0.2781 & $@ 60^{\circ}$ & $\mathrm{R}^{2}=0.79^{* * *}$ \\
\hline & & 0.2993 & $@ 90^{\circ}$ & \\
\hline & & & & Range $=40 \mathrm{~m}$, speed $=50 \mathrm{~km} / \mathrm{h}$ \\
\hline & & 0.0031 & $@ 0^{\circ}$ & \\
\hline & $\dot{\theta}$ & 0.0062 & $@ 30^{\circ}$ & $\dot{\theta}=0.0031146+0.0001014 \cdot x^{\circ}$ \\
\hline & $\theta$ & 0.0092 & $@ 60^{\circ}$ & $\mathrm{R}^{2}=0.79$ \\
\hline & & 0.0122 & $@ 90^{\circ}$ & \\
\hline & & 0.1955 & $@ 0^{\circ}$ & \\
\hline & $\tau^{-1}$ & 0.2327 & $@ 30^{\circ}$ & $\tau^{-1}=0.1955+0.0012402 \cdot x^{\circ}$ \\
\hline & $\tau^{-1}$ & 0.2699 & $@ 60^{\circ}$ & $\mathrm{R}^{2}=0.73^{* * *}$ \\
\hline & & 0.3071 & $@ 90^{\circ}$ & \\
\hline & & & & Range $=20-40 \mathrm{~m}$, speed $=50 \mathrm{~km} / \mathrm{h}$ \\
\hline & & 0.0036 & $@ 0^{\circ}$ & \\
\hline & $\dot{\theta}$ & 0.0067 & $@ 30^{\circ}$ & $\dot{\theta}=0.003635+0.00010376 \cdot x^{\circ}$ \\
\hline & $\theta$ & 0.0099 & $@ 60^{\circ}$ & $\mathrm{R}^{2}=0.80$ \\
\hline & & 0.0130 & $@ 90^{\circ}$ & \\
\hline & & 0.2171 & $@ 0^{\circ}$ & \\
\hline & $\tau^{-1}$ & 0.2455 & $@ 30^{\circ}$ & $\tau^{-1}=0.21708+0.00094742 \cdot x^{\circ}$ \\
\hline & & 0.2739 & $@ 60^{\circ}$ & $\mathrm{R}^{2}=0.62$ \\
\hline & & 0.3023 & $@ 90^{\circ}$ & \\
\hline
\end{tabular}

